\title{
Bioactive Metabolites of Endophytic fungi of Avicennia marina (Forssk.) Vierh.
}

\author{
"Sunil Kumar Deshmukh ${ }^{1,2}$, Manish Kumar Gupta ${ }^{3}$ and V.Venkateswara Sarma ${ }^{4}$ \\ ${ }^{\prime}$ TERI-Deakin Nano Biotechnology Centre, The Energy and Resources Institute (TERI), Darbari Seth Block, IHC Complex, Lodhi \\ Road, New Delhi 110003, India \\ ${ }^{2}$ Agpharm Bioinnovations LLP, Science \& Tech Entrepreneurs Park, Thapar institute of Engineering \& Technology, Patiala, \\ Punjab 147001 \\ ${ }^{3}$ SGT College of Pharmacy, SGT University, Gurugram-122505 (HR), India \\ ${ }^{4}$ Department of Biotechnology, Pondicherry University, Kalapet, Pondicherry-605014, India \\ *Corresponding author Email: sunil.deshmukh1958@gmail.com \\ (Submitted on November 15, 2020; Accepted on December 26, 2020)
}

\section{ABSTRACT}

\begin{abstract}
Endophytic fungi are microorganisms residing within the plant without causing any harm to the host plants. These fungi are known to produce diverse classes of chemical compounds with useful biological activities. Avicennia marina (Forssk.) Vierh. is a mangrove plant belonging to family Acanthaceae and used in traditional medicine. Mangrove plant $A$. marina harbors a large number of endophytic fungi which are known to produce an array of biologically active heterocyclic compounds. In the present review nearly 135 compounds reported from the endophytic fungi associated with mangrove plant $A$. marina are highlighted. These compounds were isolated from the species of genera Xylaria, Aspergillus, Penicillium, Stemphylium, Cladosporium, Phoma, and an unidentified fungus.
\end{abstract}

Keywords: Endophytic fungi, Avicennia marina, bio-active compounds, Xylaria, Penicillium

\section{INTRODUCTION}

Endophytic fungi are microorganisms residing within the plants without causing any harm to the host. These fungi are known to produce a range of chemically diverse compounds with a number of biological activities. Avicennia marina (Forssk.) Vierh. is a mangrove plant belonging to family Acanthaceae and used in traditional medicine which harbors a large number of endophytic fungi is known to produce a diverse class of heterocyclic compounds. Mangrove associated endophytic fungi are the source of various metabolites belonging to class anthraquinones, cyclic peptides, diketopiperazine, esters, isocoumarin, lactones, sesquiterpene, steroids, xanthones, and sphingolipids, xyloketals, xyloallenolides (Zhu et al., 2009; Deshmukh et al., 2015, 2018, 2020) with various biological activities such as antibacterial, antifungal, anticancer, anti-inflammatory, antioxidant, anti-angiogenesis activity, etc. In this review, we have highlighted nearly 135 compounds that are reported from species of genera Xylaria, Aspergillus, Penicillium, Stemphylium, Cladosporium, Phoma, and an unidentified fungus associated with $A$. marina (Fig. 1). The details such as location of collection of host, isolated metabolites and their biological properties are presented in table 1.

The genus Avicennia L. has five species including A. alba $\mathrm{Bl}$., A. integra N.C. Duke, A. marina, A. officinalis L. and $A$. rumphiana Hallier $\mathrm{f}$. and they all grow in mangroves. Among the different mangrove plant genera, Avicennia is the most widely distributed in the mangroves around the world (Duke, 1991). Further, from the different species in Avicennia, the plant species $A$. marina is most widely distributed (Tomlinson, 1986). A. marina is a shrub or a tree growing up to 14 meters. Three sub species of $A$. marina have been accepted including $A$. marina subsp. australasica, $A$. marina subsp. eucalyptifolia and $A$. marina subsp. marina but their distribution is less observed (Duke, 1991). Among the three

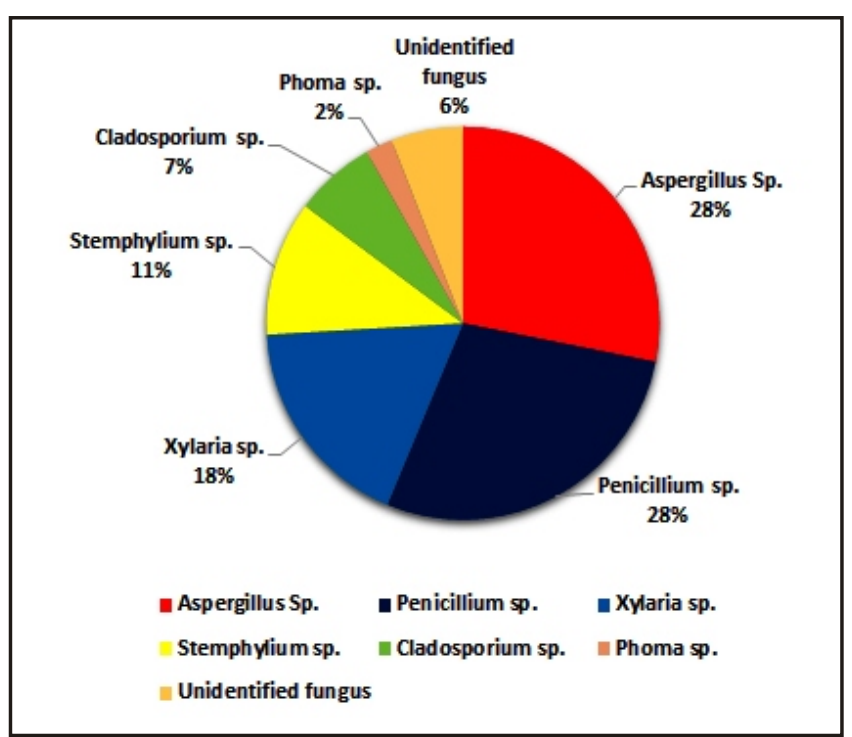

Fig. 1. Percentage distribution of bio-actives reported from various endophytic fungi of $A$. Marina

oceanic regions, $A$. marina is more widely distributed in the mangrove forests of Indian ocean region (Abdel-Wahab et al., 2020). A recent review on $A$. marina shows that on this plant alone, 149 species of marine fungi have been identified (Abdel-Wahab et al., 2020). Marine fungi are those that colonize the submerged parts of plant substrata in marine environments. The number 149 is exclusive of fungi that occur on the aerial parts of this plant. Of the 149 marine fungi identified from this host, 26 fungi were recorded only from this host (host specific) including 23 as new fungi. Since many of them are new; the host specificity may not be attributed as of now (Abdel-Wahab et al., 2020). Out of the 149 marine fungi reported from $A$. marina, only 14 marine fungal species have been investigated for their secondary metabolites. These marine fungi are known to produce novel 
bioactive compounds which possess antimicrobial, cytotoxic, phytotoxic, antimalarial and antidiabetic properties (AbdelWahab etal., 2020).

Studies on the endophytic fungal diversity in mangrove plants has gained much attention recently. Kumaresan and Suryanarayanan (2001) investigated the endophytic fungi of seven different mangrove plants in Pichavaram mangroves, Tamil Nadu, East coast of India and out of this A. marina yielded 18 fungal taxa including four sterile mycelia. A Phoma sp. is the most dominant on this host plant. Recently, the world-list of endophytic fungi has been reviewed by Rashmi et al. (2019) and they found 2770 species belonging to 877 genera. Among these Penicillium, Alternaria, Fusarium, Colletotrichum, Aspergillus and Xylaria in that order to be the most speciose genera. Some of these genera are also commonly found as endophytes on $A$. marina, belonging to Xylaria, Penicillium and Aspergillus, and most of the bioactive compounds reported from them. The fact that these few genera from $A$. marina have produced approximately 135 secondary metabolites indicates that these genera appear to be highly adaptable to all kinds of harsh-conditions and can outcompete other fungal or bacterial species in addition to protecting the host from pathogens. In bio-activity evaluation, these metabolites have shown useful pharmacological properties. Some metabolites have good potential to act as leads for the development of novel bioactive molecules with drug-like properties. In the present review, the reported bioactive metabolites from $A$. marina are discussed based on their source organisms, origin and biological properties. The summary of the bioactive metabolites and their sources are presented in Table $\mathbf{1}$ and their classification based on chemical nature is highlighted in Table 2.

\section{COMPOUNDS ISOLATED FROM XYLARIA SP.}

Five unique metabolites, xyloketals A-I (1-9), and a known compound (10) (Fig. 2) were isolated from mangrove fungus Xylaria sp. (no. 2508), obtained from the seeds of $A$. marina in Mai Po, Hong Kong. (Lin et al. 2001a; Wu et al. 2005a; b; Liu et al.; 2006; Yin et al., 2008). Xyloketal A (1) displayed the acetylcholine esterase inhibitory activity at $1.5 \times 10^{-6}$ $\mathrm{mol} / \mathrm{L}(\mathrm{p}<0.01)$ (Lin et al., 2001a). The xyloketals A (1), B (2), and F (6) displayed L-calcium channel blocking activities with inhibiting rates observed as $21.47 \%, 12.05 \%$, and $50.33 \%$, respectively at the concentration of $0.03 \mu \mathrm{M}$ (Wu et al., 2005a). Xyloketal B (2) has been implicated in the treatment for hypoxic-ischemic brain injury (Xiao et al., 2015). It also exhibited a potential for the treatment of glioblastoma which is one of the aggressive types of brain tumors (Chen et al., 2015).

Three aromatic allenic ethers xyloallenolide (11), but-2,3dienyl ether of p-hydroxycinnamic acid (12) and eucalyptene (13) (Lin et al., 2001b), and three new metabolites, named xyloester a (14), and xyloallenolide b (15), xyloketal j (16), together with a known substituted dihydrobenzofuran (17) (Fig. 2) were reported (Xu et al., 2008). A novel metabolite

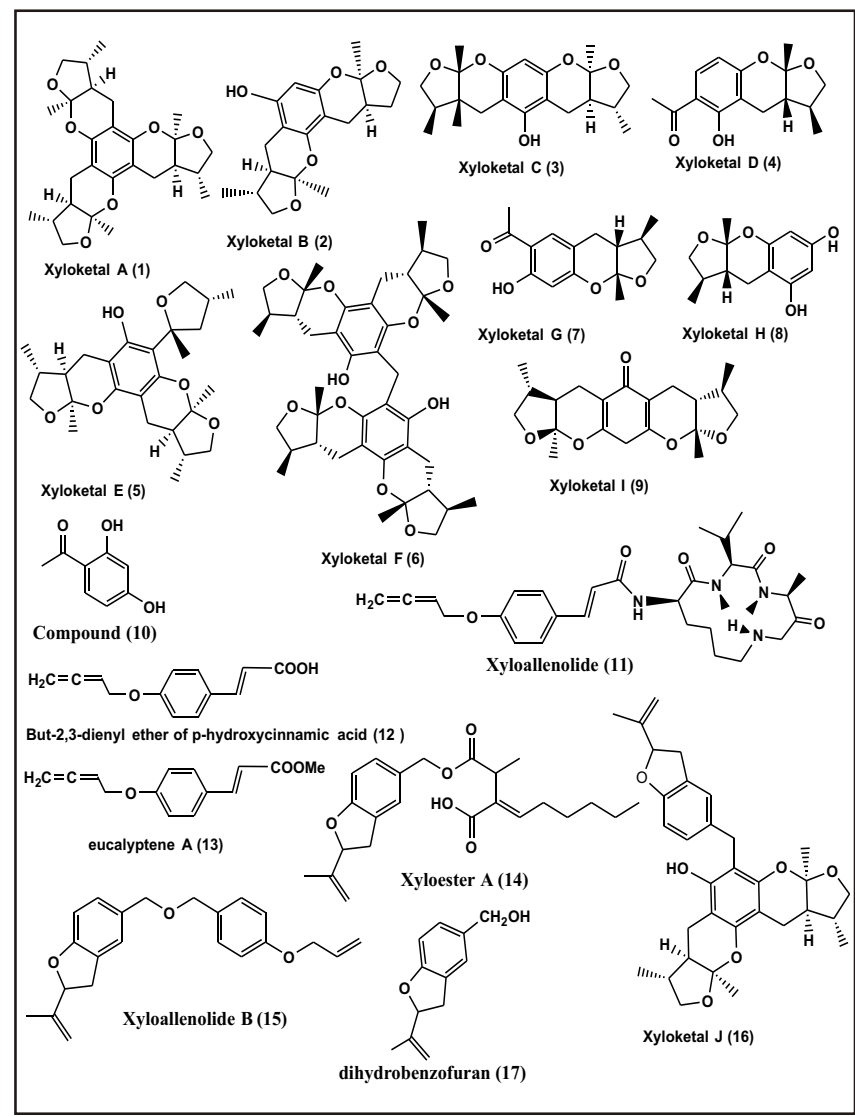

Fig. 2. Bio-actives reported from Xylaria $\mathrm{sp}$. an endophyte from A. marina (1-17).

xylopyridine A (18) and a known compound pyrocoll (19) (Xu et al., 2009), and xyloallenoide A (20) (Fig. 3), was purified from Xylaria sp. (2508) (Lu et al., 2012). Compound (18) showed a strong DNA-binding affinity toward calf thymus (CT) DNA presumably via an intercalation mechanism; thus, it is exploitable as a strong DNA-binder (Xu et al., 2009). Compound xyloallenoide A (20) induced angiogenesis in zebrafish embryos and in human endothelial cells, which was accompanied by increased phosphorylation of eNOS and Akt and NO release. Inhibition of PI3K/Akt/eNOS by LY294002 or L-NAME suppressed X13-induced angiogenesis (Lu et al., 2012).

\section{COMPOUNDS ISOLATED FROM PENICILLIUMSP.}

New polyoxygenated dihydropyrano [2,3-c]pyrrole-4, 5dione derivative called pyranonigrin $F(\mathbf{2 1})$, together with previously isolated analog, pyranonigrin A (22) (Fig. 3), were purified from Penicillium brocae MA-231, an endophytic fungus residing inside the $A$. marina. Compounds (21 and 22) exhibited potent antibacterial activity against Staphyllococcus aureus and aqua-bacteria Vibrio harveyi and V. parahaemolyticus with MIC values of $0.5 \mathrm{mg} / \mathrm{mL}$, for each strain and interestingly, appeared better than the positive control chloromycetin (with MICs 8.0, 2.0, and 128.0 $\mathrm{mg} / \mathrm{mL}$, respectively). Compounds (21 and 22) also exhibited good activity against plant pathogens Alternaria brassicae 
Table 1. Novel bioactive compounds reported from endophytic fungi associated with Avicennia marina.

\begin{tabular}{|c|c|c|c|c|c|c|}
\hline $\begin{array}{l}\text { Sr. } \\
\text { No. }\end{array}$ & Fungal strain & $\begin{array}{c}\text { Site of } \\
\text { Collection }\end{array}$ & Compounds Isolated & Biological target & $\begin{array}{c}\text { Biological active value } \\
\left(\left[\mathrm{C}_{50} / \mathrm{ED}_{50}\right)\right.\end{array}$ & Reference \\
\hline 1. & $\begin{array}{l}\text { Xylaria sp. } \\
\text { (no. 2508) }\end{array}$ & $\begin{array}{l}\text { Mai Po, Hong } \\
\text { Kong }\end{array}$ & Xyloketal A (1), & acetylcholine esterase. & $1.5 \times 10-6 \mathrm{~mol} / \mathrm{L}$ & Lin et al., 2001a \\
\hline 2. & $\begin{array}{l}\text { Xylaria sp. } \\
\text { (no. } 2508)\end{array}$ & $\begin{array}{l}\text { Mai Po, Hong } \\
\text { Kong }\end{array}$ & Xyloketal B (2), & & & Lin et al., 2001a \\
\hline 3. & $\begin{array}{l}\text { Xylaria sp. } \\
\text { (no. 2508) }\end{array}$ & $\begin{array}{l}\text { Mai Po, Hong } \\
\text { Kong }\end{array}$ & Xyloketal C (3), & & & Lin et al., 2001a \\
\hline 4. & $\begin{array}{l}\text { Xylaria sp. } \\
\text { (no. 2508) }\end{array}$ & $\begin{array}{l}\text { Mai Po, Hong } \\
\text { Kong }\end{array}$ & Xyloketal D (4), & & & Lin et al., 2001a \\
\hline 5. & $\begin{array}{l}\text { Xylaria sp. } \\
\text { (no. 2508) }\end{array}$ & $\begin{array}{l}\text { Mai Po, Hong } \\
\text { Kong }\end{array}$ & Xyloketal E (5), & & & Lin et al., 2001a \\
\hline 6. & $\begin{array}{l}\text { Xylariasp. } \\
\text { (no. 2508) }\end{array}$ & $\begin{array}{l}\text { Mai Po, Hong } \\
\text { Kong }\end{array}$ & Xyloketal F (6), xyloketals A (1), B (2) & L-calcium channel blocking activity & $\begin{array}{l}\text { Inhibition rates were } 21.47 \% \text {, } \\
12.05 \% \text {, and } 50.33 \% \text { at } 0.03 \\
\mu \mathrm{M} / \mathrm{L} \text { concentration }\end{array}$ & Wu et al. $2005 \mathrm{a}$ \\
\hline 7. & $\begin{array}{l}\text { Xylaria sp. } \\
\text { (no. 2508) }\end{array}$ & $\begin{array}{l}\text { Mai Po, Hong } \\
\text { Kong }\end{array}$ & xyloketal G (7), & & & Wu et al. $2005 \mathrm{~b}$ \\
\hline 8. & $\begin{array}{l}\text { Xylaria sp. } \\
\text { (no. 2508) }\end{array}$ & $\begin{array}{l}\text { Mai Po, Hong } \\
\text { Kong }\end{array}$ & Xyloketal H (8), & & & $\begin{array}{l}\text { Liu et al., 2006, } \\
\text { Yin et al., 2008 }\end{array}$ \\
\hline 9. & $\begin{array}{l}\text { Xylaria sp. } \\
\text { (no. 2508) }\end{array}$ & $\begin{array}{l}\text { Mai Po, Hong } \\
\text { Kong }\end{array}$ & Xyloketal I (9) & & & \\
\hline 10. & $\begin{array}{l}\text { Xylaria sp. } \\
\text { (no. 2508) }\end{array}$ & $\begin{array}{l}\text { Mai Po, Hong } \\
\text { Kong }\end{array}$ & Compound (10) & & & Lin et al., 2001a \\
\hline 11. & $\begin{array}{l}\text { Xylaria sp. } \\
\text { (no. 2508) }\end{array}$ & $\begin{array}{l}\text { Mai Po, Hong } \\
\text { Kong }\end{array}$ & $\begin{array}{l}\text { Xyloallenolide (11) But-2,3-dienyl ether of } \\
\text { p-hydroxycinnamic acid (12) and } \\
\text { Eucalyptene (13) }\end{array}$ & & & Lin et al., 2001b \\
\hline 12. & $\begin{array}{l}\text { Xylaria sp. } \\
\text { (no. 2508) }\end{array}$ & $\begin{array}{l}\text { Mai Po, Hong } \\
\text { Kong }\end{array}$ & $\begin{array}{l}\text { Xyloester A (14), Xyloallenolide B (15), } \\
\text { Xyloketal J (16), Dihydrobenzofuran (17) }\end{array}$ & & & Xu et al., 2008 \\
\hline \multirow[t]{2}{*}{13.} & \multirow[t]{2}{*}{$\begin{array}{l}\text { Xylaria sp. } \\
\text { (no. 2508) }\end{array}$} & \multirow[t]{2}{*}{$\begin{array}{l}\text { Mai Po, Hong } \\
\text { Kong }\end{array}$} & Xylopyridine A (18), & $\begin{array}{l}\text { Strong DNA-binding affinity toward } \\
\text { calf thymus (CT) DNA presumably via } \\
\text { an intercalation mechanism, thus it is } \\
\text { exploitable as a strong DNA-binders }\end{array}$ & & \multirow[t]{2}{*}{ Xu et al., 2009} \\
\hline & & & Pyrocoll (19), & & & \\
\hline 14. & $\begin{array}{l}\text { Xylaria sp. } \\
\text { (no. 2508) }\end{array}$ & $\begin{array}{l}\text { Mai Po, Hong } \\
\text { Kong }\end{array}$ & Xyloallenoide A (20), & $\begin{array}{l}\text { Inhibition of PI3K/Akt/eNOS by } \\
\text { LY294002 }\end{array}$ & $\begin{array}{l}\text { Induces angiogenesis in zebra } \\
\text { fish embryos and in human } \\
\text { endothelial cells }\end{array}$ & Lu et al., 2012 \\
\hline \multirow[t]{2}{*}{15.} & \multirow{2}{*}{$\begin{array}{l}\text { Penicillium } \\
\text { brocae MA- } \\
231\end{array}$} & & $\begin{array}{l}\text { Pyranonigrin F (21), Pyranonigrin A (22) } \\
\text { Positive control chloromycetin }\end{array}$ & $\begin{array}{l}\text { S. aureus and aqua-bacteria Vibrio } \\
\text { harveyi and V. parahaemolyticus }\end{array}$ & $\begin{array}{l}\text { MIC, } 0.5 \mu \mathrm{g} / \mathrm{mL} \text { each } \\
\text { MICs } 8.0,2.0 \text {, and } 128.0 \mu \mathrm{g} / \mathrm{mL} \text { ) }\end{array}$ & \multirow[t]{2}{*}{$\begin{array}{l}\text { Meng et al., } \\
2015 \mathrm{a}\end{array}$} \\
\hline & & & $\begin{array}{l}\text { Pyranonigrin F (21), Pyranonigrin A (22) } \\
\text { Positive control bleomycin }\end{array}$ & $\begin{array}{l}\text { Alternaria brassicae and Colletotrichum } \\
\text { gloeosprioides }\end{array}$ & $\begin{array}{l}\text { MICs, } 0.5 \mu \mathrm{g} / \mathrm{mL} \text { for each strain } \\
\text { MICs } 32.0 \text { and } 4.0 \mu \mathrm{g} / \mathrm{mL}\end{array}$ & \\
\hline \multirow[t]{7}{*}{16.} & \multirow{7}{*}{$\begin{array}{l}\text { Penicillium } \\
\text { brocae MA- } \\
231\end{array}$} & & Penicibrocazines A (23) & & & \multirow{7}{*}{$\begin{array}{l}\text { Meng et al., } \\
2015 \mathrm{~b}\end{array}$} \\
\hline & & & $\begin{array}{l}\text { Penicibrocazines B-D (24-26), Phomazine B } \\
\text { (28), }\end{array}$ & Staphylococcus aureus, & $\begin{array}{l}\text { MIC, } 32.0,0.25,8.0 \text {, and } 0.25 \\
\mu \mathrm{g} / \mathrm{mL} \text {. }\end{array}$ & \\
\hline & & & Positive control, chloromycetin & & $\mathrm{MIC}, 4.0 \mu \mathrm{g} / \mathrm{mL}$ & \\
\hline & & & Penicibrocazine C (25) & Micrococcus luteus & MIC, $0.25 \mu \mathrm{g} / \mathrm{mL}$ & \\
\hline & & & Positive control, chloromycetin & \multirow{3}{*}{ Gaeumannomyces graminis } & MIC, $2.0 \mu \mathrm{g} / \mathrm{mL}$ & \\
\hline & & & $\begin{array}{l}\text { Penicibrocazines B (24), } \\
\text { Penicibrocazines D (26), } \\
\text { Penicibrocazines E (27), } \\
\text { Phomazine B (28), }\end{array}$ & & $\begin{array}{l}\mathrm{MIC}, 0.25,8.0,0.25 \text {, and } 64.0 \\
\mu \mathrm{g} / \mathrm{mL}\end{array}$ & \\
\hline & & & Positive control amphotericin B & & MIC, $16.0 \mu \mathrm{g} / \mathrm{mL}$ & \\
\hline 17. & $\begin{array}{l}\text { Penicillium } \\
\text { brocae MA- } \\
231\end{array}$ & & Brocazines A, B, E, F $(\mathbf{2 9}, \mathbf{3 0}, \mathbf{3 3}, \mathbf{3 4})$, & $\begin{array}{l}\text { Du145, HeLa, HepG2, MCF-7, NCI- } \\
\text { H460, SGC-7901, SW1990, SW480, } \\
\text { and U251 cell lines }\end{array}$ & $\begin{array}{l}\mathrm{IC}_{50} \text { values ranging from } 0.89 \text { to } \\
9.0 \mu \mathrm{M} \text {. }\end{array}$ & \multirow[t]{4}{*}{ Meng et al., 2014} \\
\hline \multirow[t]{3}{*}{18.} & \multirow{3}{*}{$\begin{array}{l}\text { Penicillium } \\
\text { brocae MA- } \\
231\end{array}$} & & Brocazines A, B $(\mathbf{2 9}, \mathbf{3 0})$, & SW480 tumor cell line & $\mathrm{IC}_{50}, 2.0$ and $1.2 \mu \mathrm{M}$ & \\
\hline & & & $\begin{array}{l}\text { Brocazines F (34) } \\
\text { Brocazines C-D (31, 32), Epicorazine A }\end{array}$ & DU145 and NCI-H460 cell lines, & $\mathrm{IC}_{50} 1.7$ and $0.89 \mu \mathrm{M}$, & \\
\hline & & & $\begin{array}{l}\text { Brocazınes C-D }(31,32) \text {, Epicorazine A } \\
(\mathbf{3 5})\end{array}$ & - & - & \\
\hline \multirow[t]{9}{*}{19.} & \multirow[t]{9}{*}{$\begin{array}{l}\text { Penicillium } \\
\text { brocae MA- } \\
231\end{array}$} & & 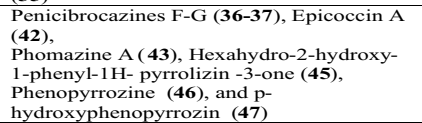 & & & \multirow[t]{9}{*}{ Meng et al. ,2017 } \\
\hline & & & $\begin{array}{l}\text { Brocapyrrozins A(40), } \\
\text { 4-Hydroxy-3-phenyl-1 } \mathrm{H} \text {-pyrrol2(5H)-one } \\
\text { (44), }\end{array}$ & S. aureus & $\begin{array}{l}\text { MIC values of } 0.125 \text { and } 0.5 \\
\mu \mathrm{g} / \mathrm{mL} \text {, }\end{array}$ & \\
\hline & & & Positive control, chloromycetin & & MIC value of $0.5 \mu \mathrm{g} / \mathrm{mL}$ & \\
\hline & & & Penicibrocazines H, I (38-39), & V. harveyi & $\begin{array}{l}\text { MIC values of } 16.0 \text { and } 32.0 \\
\mu \mathrm{g} / \mathrm{mL}\end{array}$ & \\
\hline & & & Positive control chloromycetin & & MIC value of $4 \mu \mathrm{g} / \mathrm{mL}$ & \\
\hline & & & Penicibrocazines H (38), & $\begin{array}{l}\text { E. coli, A. hydrophilia and } V \text {. } \\
\text { parahaemolyticus }\end{array}$ & $\begin{array}{l}\mathrm{MIC}, 16.0,32.0 \text {, and } 16.0 \mu \mathrm{g} / \\
\mathrm{mL}\end{array}$ & \\
\hline & & & Positive control, chloromycetin & & $\mathrm{MIC}, 2.0,4.0,2.0 \mu \mathrm{g} / \mathrm{mL}$ & \\
\hline & & & $\begin{array}{l}\text { Brocapyrrozins A and B (40-41), 4- } \\
\text { Hydroxy-3-phenyl-1H-pyrrol2(5H)-one (44) }\end{array}$ & F. xysporum & $\begin{array}{l}\mathrm{MIC}, 0.2564 .0 \text { and } 0.125 \mu \mathrm{g} / \\
\mathrm{mL} \text {, }\end{array}$ & \\
\hline & & & Positive control zeocin & & MIC, $0.5 \mu \mathrm{g} / \mathrm{mL}$ & \\
\hline 20. & $\begin{array}{l}\text { Penicillium } \\
\text { brocae MA- }\end{array}$ & $\begin{array}{l}\text { Hainan Island, } \\
\text { China }\end{array}$ & Spirobrocazines A (48) & $\begin{array}{l}\text { Escherichia coli, S. aureus and Vibrio } \\
\text { harveyi, }\end{array}$ & $\begin{array}{l}\mathrm{MIC}, 32.0,16.0, \text { and } 64.0 \\
\mu \mathrm{g} / \mathrm{mL} \text {. }\end{array}$ & Meng et al., 2016 \\
\hline & & & Positive control chloromycetin & & MIC, $2.0,0.5$, and $2.0 \mu \mathrm{g} / \mathrm{mL}$ & \\
\hline & & & Spirobrocazine B (49) & & & \\
\hline & & & Spirobrocazine C (50) & A2780 cells & $\mathrm{IC}_{50}, 59 \mu \mathrm{M}$ & \\
\hline & & & Spirobrocazine C (50) & $\begin{array}{l}\text { E. coli, Aeromonas hydrophilia, and } V \text {. } \\
\text { harveyi, }\end{array}$ & MIC, $32.0 \mu \mathrm{g} / \mathrm{mL}$ & \\
\hline & & & Brocazine G (51) & $\mathrm{A} 2780$ and $\mathrm{A} 2780 \mathrm{CisR}$ & $\mathrm{IC}_{50}, 664$ and $661 \mathrm{nM}$ & \\
\hline & & & Positive control cisplatin & & $\mathrm{IC}_{50}, 1.67$ and $12.63 \mu \mathrm{M}$ & \\
\hline & & & Brocazine G (51) & Staphylococcus aureus & \begin{tabular}{|l|} 
MIC, $0.25 \mu \mathrm{g} / \mathrm{mL}$ \\
\end{tabular} & \\
\hline & & & Positive control, chloromycetin & & $\mathrm{MIC}, 0.5 \mu \mathrm{g} / \mathrm{mL}$ & \\
\hline 21. & $\begin{array}{l}\text { Penicillium } \\
\text { sp.FJ-1 }\end{array}$ & & $\begin{array}{l}\text { (Z)-7,4'-dimethoxy-6-hydroxy-aurone-4-O- } \\
\beta \text { - glucopyranoside (52), }\end{array}$ & $\begin{array}{l}\text { Candida sp., inhibit extracellular } \\
\text { phospholipase secretion }\end{array}$ & & Song et al., 2015 \\
\hline 22. & $\begin{array}{l}\text { Penicillium } \\
\text { citrinum }\end{array}$ & & $\begin{array}{l}\text { (Z)-7,4'-dimethoxy-6-hydroxy-aurone-4-O- } \\
\beta \text {-glucopyranoside (52) }\end{array}$ & & & Liu et al., 2015a \\
\hline & & & 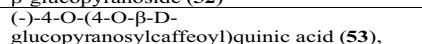 & Chemoreversal activity & $\begin{array}{l}\text { Inhibiting P-glycoprotein efflux } \\
\text { pump function }\end{array}$ & \\
\hline 23. & $\begin{array}{l}\text { Penicillium } \\
\text { sp.FJ-1 }\end{array}$ & Fujian, China & $\begin{array}{l}\text { 4-(2,'3'-dihydroxy-3'-methyl-butanoxy)- } \\
\text { phenethanol (54), }\end{array}$ & Tca8113 and MG-63 cells & $\mathrm{IC}_{50}, 26$ and $35 \mu \mathrm{M}$ & Zheng et al., 2014 \\
\hline & & & 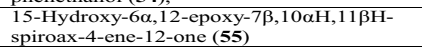 & $\begin{array}{l}\text { Tca8113, MG-63 and the normal liver } \\
\text { cell line WRL-68 }\end{array}$ & $\mathrm{IC}_{50}, 10,55$ and $58 \mu \mathrm{M}$ & \\
\hline & & & Taxol, the positive control & Tca8 113 and MG -63 cell lines & $\mathrm{IC}_{50}, 46$ and $10 \mathrm{nM}$ & \\
\hline 24. & $\begin{array}{l}\text { Penicillium } \\
\text { brocae MA- } \\
192\end{array}$ & $\begin{array}{l}\text { Hainan Island, } \\
\text { China }\end{array}$ & $\begin{array}{l}\text { (10R, 14R)-10-Hydroxydihydroresorcylide } \\
(56), \text { brocaketone A (57), brocaketone D } \\
(\mathbf{5 8})\end{array}$ & DPPH assay & $\mathrm{IC}_{50}, 14.4,5.9$, and $16.3 \mu \mathrm{g} / \mathrm{ml}$ & Zhang et al., 2015 \\
\hline & & & Positive control BHT & & $\mathrm{IC}_{50}, 18.5 \mu \mathrm{g} / \mathrm{ml}$ & \\
\hline 25. & $\begin{array}{l}\text { Aspergillus } \\
\text { versicolor }\end{array}$ & $\begin{array}{l}\text { Port Safaga, } \\
\text { Red Sea } \\
\text { Governorate, } \\
\text { Egypt }\end{array}$ & Allantopyrone E (59) & HeLa cells & $\mathrm{IC}_{50}, 50.97 \mu \mathrm{M}$ & Elsbaey et al.2020 \\
\hline
\end{tabular}


cont.

\begin{tabular}{|c|c|c|c|c|c|c|}
\hline $\begin{array}{l}\text { Sr. } \\
\text { No. }\end{array}$ & Fungal strain & $\begin{array}{c}\text { Site of } \\
\text { Collection }\end{array}$ & Compounds Isolated & Biological target & $\begin{array}{c}\text { Biological active value } \\
\left(\mathrm{IC}_{50} / \mathbf{E D}_{50}\right)\end{array}$ & Reference \\
\hline \multirow[t]{6}{*}{26.} & \multirow{6}{*}{$\begin{array}{l}\text { Aspergillus } \\
\text { niger MA- } \\
132\end{array}$} & & $\begin{array}{l}\text { Nigerapyrones A-C (60-62), Nigerapyrones } \\
\text { F-H (65-67), Asnipyrones B (68) }\end{array}$ & & & \multirow[t]{6}{*}{ Liu et al,. 2011} \\
\hline & & & Nigerapyrone B (61), & HepG2 cell line & $\mathrm{IC}_{50}, 62 \mu \mathrm{M}$ & \\
\hline & & & Nigerapyrone D (63) & MCF-7, HepG2, and A549 cell lines & $\mathrm{IC}_{50}, 121,81$, and $81 \mu \mathrm{M}$ & \\
\hline & & & Nigerapyrone E (64) & $\begin{array}{l}\text { SW1990, MDA-MB-231, A549 MCF- } \\
\text { 7, HepG2, Du145, NCI-H460, and } \\
\text { MDA-MB-231 cell lines }\end{array}$ & $\begin{array}{l}\mathrm{IC}_{50,38,48,43,105,86,86,43,} \\
\text { and } 48 \mu \mathrm{M},\end{array}$ & \\
\hline & & & Positive control, fluorouracil & $\begin{array}{l}\text { A549, HepG2, DU145,MCF-7, } \\
\text { SW1990, NCI-H460, and MDA-MB- } \\
231 \text { cell lines }\end{array}$ & $\begin{array}{l}\mathrm{IC}_{50,} 52,109,3.3,31,121,8.5 \\
\text { and } 59 \mu \mathrm{M}\end{array}$ & \\
\hline & & & Asnipyrone A (69) & A549 cell line & $\mathrm{IC}_{50 .}, 62 \mu \mathrm{M}$ & \\
\hline 27. & $\begin{array}{l}\text { Aspergillus } \\
\text { niger MA- } \\
132\end{array}$ & & $\begin{array}{l}\text { Nigerasterols A(70), B (71), Malformins } \\
A_{1}(\mathbf{7 2}), C(\mathbf{7 3})\end{array}$ & HL60 and A549 cell lines & & Liu et al., 2013 \\
\hline \multirow[t]{2}{*}{28.} & \multirow[t]{2}{*}{$\begin{array}{l}\text { Aspergillus } \\
\text { versicolor }\end{array}$} & \multirow[t]{2}{*}{$\begin{array}{l}\text { 17 K Safaga, } \\
\text { Red Sea, Egypt }\end{array}$} & $\begin{array}{l}\text { Anthcolorin G (74), (7R,8R)-8- } \\
\text { hydroxysydowic acid (76), (7S,10S)-10- } \\
\text { hydroxy-sydowic acid (77), (7S,11R)-12- } \\
\text { hydroxy-sydowic acid (78), (7S,11R)-12- } \\
\text { Acetoxy-sydowic acid (79), (7R,8R)-1,8- } \\
\text { epoxy-11-hydroxy-sydonic acid (80), 7- } \\
\text { deoxy-7,14-didehydro -11-hydroxysydonic } \\
\text { acid (81), (7R)-11-hydroxy-sydonic acid } \\
\text { methyl ester (84), 3-hydroxy-4-(1-oxo- } \\
\text { ethane) benzoic acid (85), (S)- sydowic acid } \\
\text { (86), (7R,10R)-iso-10-hydroxy-sydowic } \\
\text { acid (87), Engyodontiumone J (88), } \\
\text { engyodontiumone I (89), (E)-7-deoxy-7,8- } \\
\text { didehydro12-hydroxy-sydonic acid (90), } \\
\text { (7R)-11-hydroxysydonic acid (91), an } \\
\text { epimeric mixture of (7R,11R), (7R,11S)-12- } \\
\text { acetoxy sydonic acid, (92), 12-acetoxy-1- } \\
\text { deoxy-sydonic (93), Macrosporin (95), } \\
\text { Ergostrol peroxide (96) }\end{array}$ & & & \multirow[t]{2}{*}{$\begin{array}{l}\text { Elsbaey et al., } \\
2019\end{array}$} \\
\hline & & & $\begin{array}{l}\text { Anthcolorin H (75), 7-deoxy-7,14- } \\
\text { didehydro-12-acetoxy-sydonic acid (82), } \\
\text { (E)-7-deoxy-7,8-didehydro-12- acetoxy- } \\
\text { sydonic acid (83), Diorcinol } \mathbf{( 9 4 )}\end{array}$ & Hela cells & $\mathrm{IC}_{50,} 43.7,83.8,53.5,83.8 \mu \mathrm{M}$ & \\
\hline \multirow[t]{4}{*}{29.} & \multirow[t]{4}{*}{$\begin{array}{l}\text { Stemphylium } \\
\text { globuliferum }\end{array}$} & & $\begin{array}{l}\text { Altersolanol Q (97), 10-methylaltersolanol } \\
\text { Q (98), Alterporriol X (99), } \\
\text { Dihydroaltersolanol B (100) and C (101), } \\
\text { Altersolanol A (102), B (103), N (104), 1- } \\
\text { hydroxy3-methoxy-6-methylanthraquinone } \\
\text { (105), Macrosporin (95), Altechromone A } \\
(\mathbf{1 0 6}) \text {, Alterporriol D (107), E (108), R } \\
\text { (109), V (110), and W (111) }\end{array}$ & & & $\begin{array}{l}\text { Moussa et al, } \\
2016\end{array}$ \\
\hline & & & $\begin{array}{l}\text { Dihydroaltersolanol C (101), Altersolanol } \\
\mathrm{A},(\mathbf{1 0 2}) \mathrm{B},(\mathbf{1 0 3}) \text {, Alterporriol E (108), }\end{array}$ & L5178Y mouse lymphoma cell line & $\mathrm{IC}_{50,3.4,2.53,3.78 \text {, and } 6.9, \mu \mathrm{m}}$ & $\begin{array}{l}\text { Debbab et al. } \\
\text {,2009; Liu et al., } \\
2015 \text { b }\end{array}$ \\
\hline & & & Altersolanol N (104) & L5178Y cells & $\begin{array}{l}\mathrm{IC}_{50} \text { values in the low micro- } \\
\text { molar range }\end{array}$ & $\begin{array}{l}\text { Debbab et al., } \\
2012\end{array}$ \\
\hline & & & altersolanol A (102) & 34 human cancer cell lines & $\begin{array}{l}\text { Mean IC } \mathrm{IC}_{50}\left(\mathrm{IC}_{70}\right) \text { values of } 0.005 \\
\mu \mathrm{g} / \mathrm{mL}(0.024 \mu \mathrm{g} / \mathrm{mL})\end{array}$ & $\begin{array}{l}\text { Mishra et al., } \\
2015\end{array}$ \\
\hline \multirow[t]{3}{*}{30.} & \multirow[t]{3}{*}{$\begin{array}{l}\text { Cladosporium } \\
\text { oxysporum }\end{array}$} & \multirow[t]{3}{*}{$\begin{array}{l}\text { Hainan } \\
\text { Province, China }\end{array}$} & $\begin{array}{l}\text { Thiocladospolides F-J (112-116), } \\
\text { Pandangolide } 3 \text { (117), Thiocladospolide A } \\
\text { (118), Seco-secopatulolide C (119), and Iso- } \\
\text { cladospolide B (120) }\end{array}$ & Edwardsiella tarda and E. ictarda & $\begin{array}{l}\text { MIC values ranging from } 4 \text { to } 32 \\
\mu \mathrm{g} / \mathrm{mL} \text {. }\end{array}$ & \multirow[t]{3}{*}{ Wang et al., 2020} \\
\hline & & & Thiocladospolide G (113) & E. tarda & MIC, $4 \mu \mathrm{g} / \mathrm{mL}$ & \\
\hline & & & Iso-cladospolide B $120(\mathbf{1 2 0})$ & Cytospora mandshurica & MIC, $8 \mu \mathrm{g} / \mathrm{mL}$ & \\
\hline 31 & $\begin{array}{l}\text { Phoma sp. } \\
\text { SK3RW1M }\end{array}$ & $\begin{array}{l}\text { Shankou } \\
\text { mangrove, } \\
\text { Guangxi, P. R. } \\
\text { China }\end{array}$ & $\begin{array}{l}\text { 1,8-dihydroxy-10-methoxy-3- } \\
\text { methyldibenzo[b,e]oxepine-6,11-dione } 121 \\
\text { (121), 1-hydroxy-8-(hydroxymethyl)-6- } \\
\text { methoxy -3-methyl -9H- xanthen-9-one } 122 \\
\text { (122), 1-hydroxy-8-(hydroxymethyl)-3- } \\
\text { methoxy-6-methyl-9H-xanthen -9-one } 123 \\
\text { (123), }\end{array}$ & Cytotoxic & Inactive & Pan et al., 2010 \\
\hline 32 & $\begin{array}{l}\text { Xylaria sp. } \\
\text { (No. 2524) }\end{array}$ & & $\begin{array}{l}\text { Cyclo-(L-Phe-L-Leu1-L-Leu2-L-Leu3-L- } \\
\text { Ile) (124), (3S,4R)-dihydroxy-(6S)-undecyl- } \\
\alpha \text {-pyranone }(\mathbf{1 2 5})\end{array}$ & Bel-7402, NCI-4460 and L-02 cell lines & Poorly active & Li et al., 2004 \\
\hline 33. & $\begin{array}{l}\text { Endophyte } \\
\text { No. } 2106\end{array}$ & Hong Kong & $\begin{array}{l}2106 \mathrm{~A}(\mathbf{1 2 6}), \text { Cyclo-(N-MeVal-N-MeAla) } \\
(\mathbf{1 2 7})\end{array}$ & - & - & Wang et al., 2008 \\
\hline \multirow[t]{2}{*}{34.} & \multirow[t]{2}{*}{$\begin{array}{l}\text { Unidentified } \\
\text { endophytic } \\
\text { fungus }\end{array}$} & \multirow[t]{2}{*}{ Oman } & $\begin{array}{l}\text { Farinomalein (128), Farinomaleins B-E } \\
\text { (129-132), (3R)-5,7-dihydroxy-3- } \\
\text { methylisoindolin-1-one. (133) }\end{array}$ & & & \multirow[t]{2}{*}{$\begin{array}{l}\text { El Amrani et al., } \\
2012\end{array}$} \\
\hline & & & Farinomaleins B (129) & & $\mathrm{IC}_{50 .} 4.4 \mu \mathrm{g} / \mathrm{mL}$ & \\
\hline \multirow[t]{2}{*}{35.} & \multirow{2}{*}{$\begin{array}{l}\text { Endophytic } \\
\text { fungus (No. } \\
\text { ZH19) }\end{array}$} & \multirow{2}{*}{$\begin{array}{l}\text { Dong Sai of the } \\
\text { South China } \\
\text { Sea coast }\end{array}$} & $\begin{array}{l}\text { 1,7-Dihydroxy-2-methoxy-3-(3-methylbut- } \\
\text { 2-enyl) -9H-xanthen -9-one (134) }\end{array}$ & KB and KBV200 cells & $\begin{array}{l}\mathrm{IC}_{50,}, 20 \text { and } \\
30 \mu \mathrm{M}\end{array}$ & \multirow[t]{2}{*}{ Huang et al,. 2010} \\
\hline & & & $\begin{array}{l}\text { 1-Hydroxy-4,7-dimethoxy-6-(3-oxobutyl) - } \\
\text { 9H-xanthen9-one (135). }\end{array}$ & KB and KBV200 cells & $\mathrm{IC}_{50}, 35$ and $41 \mu \mathrm{M}$ & \\
\hline
\end{tabular}

and Colletotrichum gloeosprioides with MICs of $0.5 \mathrm{mg} / \mathrm{mL}$, which was better than positive control bleomycin (with MICs 32.0 and $4.0 \mathrm{mg} / \mathrm{mL}$, respectively) (Meng et al., 2015a).

Five new sulfide diketopiperazine derivatives, namely, penicibrocazines A-E (23-27) (Fig. 3), along with previously isolated congener phomazine B (28) (Fig. 4), were purified from Penicillium brocae MA-231. Compounds (24-26 and 28) displayed antibacterial activity against Staphylococcus aureus, with MIC values of $32.0,0.25,8.0$, and $0.25 \mu \mathrm{g} / \mathrm{mL}$, 
respectively, (positive control, chloromycetin, $\mathrm{MIC}=4.0$ $\mu \mathrm{g} / \mathrm{mL}$ ). Compound (25) also showed activity against Micrococcus luteus with MIC value of $0.25 \mu \mathrm{g} / \mathrm{mL}$, which was better than that of the positive control, chloromycetin $(\mathrm{MIC}=2.0 \mu \mathrm{g} / \mathrm{mL})$. In addition, compounds $(\mathbf{2 4}, \mathbf{2 6}, 27$ and 28) displayed activity against plant pathogen Gaeumannomyces graminis with MIC values of $0.25,8.0$, 0.25 , and $64.0 \mu \mathrm{g} / \mathrm{mL}$, respectively, (positive control amphotericin B, MIC $=16.0 \mu \mathrm{g} / \mathrm{mL}$ ) (Meng et al., 2015b).

Six new disulfide-bridged diketopiperazine derivatives, brocazines A-F (29-34), together with previously isolated analog epicorazine A (35) (Fig. 4), were purified from Penicillium brocae MA-231. Compounds (29, 30, 33 and 34) exhibited cytotoxicity with IC50 values ranging from 0.89 to $9.0 \mu \mathrm{M}$ against the Du145, HeLa, HepG2, MCF-7, NCIH460, SGC-7901, SW1990, SW480, and U251 cell lines. Compounds (29 and 30) exhibited good activity against the SW480 cells, with $\mathrm{IC}_{50}$ values of 2.0 and $1.2 \mu \mathrm{M}$, respectively. Compound (34) displayed potent activity against the DU145 and NCI-H460 cells, with IC50 values of 1.7 and $0.89 \mu \mathrm{M}$, respectively (Meng et al., 2014).

Four new thiodiketopiperazine alkaloids, penicibrocazines FI (36-39), along with two new nitrogen-containing phydroxyphenopyrrozin derivatives brocapyrrozins $\mathrm{A}$ and $\mathrm{B}$ (40-41) as well as six known alkaloids epicoccin A (42), phomazine A (43), 4-hydroxy-3-phenyl-1H-pyrrol2(5H)-one (44), hexahydro-2-hydroxy-1-phenyl-1H-pyrrolizin-3-one

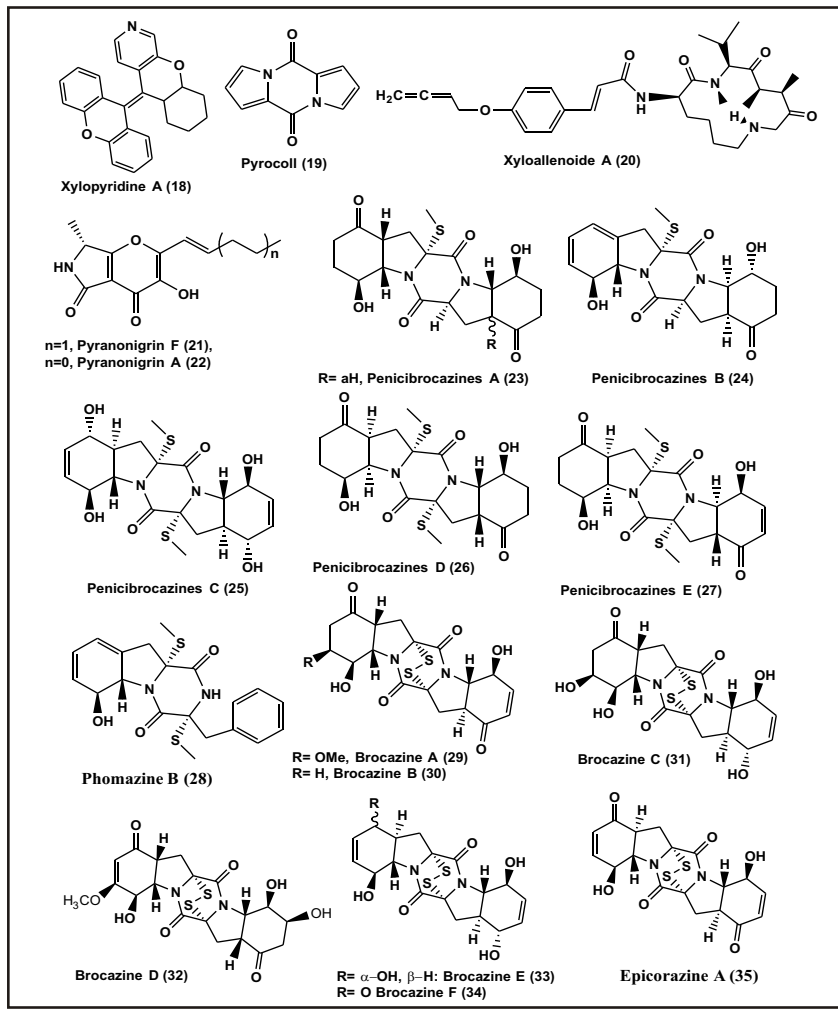

Fig. 3. Bio-actives reported from Xylaria sp. (18-20) and Penicillium sp. (21-35) an endophyte from $A$. marina.
(45), phenopyrrozine (46) (Fig. 4), and p-hydroxyphenopyrrozin (47) (Fig. 5), were purified from mangrovederived endophytic fungus $P$. brocae MA-231 using OSMAC (one strain-many compounds) method. Compounds (40) and (44) exhibited good antibacterial activity against S. aureus with MIC values of 0.125 and $0.5 \mu \mathrm{g} / \mathrm{mL}$, respectively (positive control, chloromycetin, $\mathrm{MIC}=0.5 \mu \mathrm{g} / \mathrm{mL}$ ). While compounds (38) and (39) displayed activity against $V$. harveyi with MIC values of 16.0 and $32.0 \mu \mathrm{g} / \mathrm{mL}$, respectively, (positive control chloromycetin, MIC $=4 \mu \mathrm{g} / \mathrm{mL}$ ). Compound (38) also displayed antibacterial activity against Escherichia coli, Aeromonas hydrophilia and Vibrio parahaemolyticus with MIC values of 16.0, 32.0, and 16.0 $\mu \mathrm{g} / \mathrm{mL}$, respectively, (positive control, chloromycetin, $\mathrm{MIC}=$ 2.0, 4.0, $2.0 \mu \mathrm{g} / \mathrm{mL})$. Compounds (40, 44 and 41) exhibited good antifungal activity against Fusarium oxysporum MIC values of $0.25,0.125$ and $64.0 \mu \mathrm{g} / \mathrm{mL}$ respectively, while positive control zeocin exhibited antifungal activity with MIC value of $0.5 \mu \mathrm{g} / \mathrm{mL}$ (Meng et al., 2017).

Four new diketopiperazines including spirobrocazines A-C (48-50) and brocazine G (51) (Fig. 5), were purified from Penicillium brocae MA-231 using the one strain many compounds (OSMAC) approach. Compound (51) displayed potent cytotoxic activity against A2780 and 2780 CisR cell lines, with $\mathrm{IC}_{50}$ values of 6.64 and $6.61 \mu \mathrm{M}$, respectively, which was found better than cisplatin (positive control) with $\mathrm{IC}_{50}$, value of 1.67 and $12.63 \mu \mathrm{M}$, respectively. In addition,

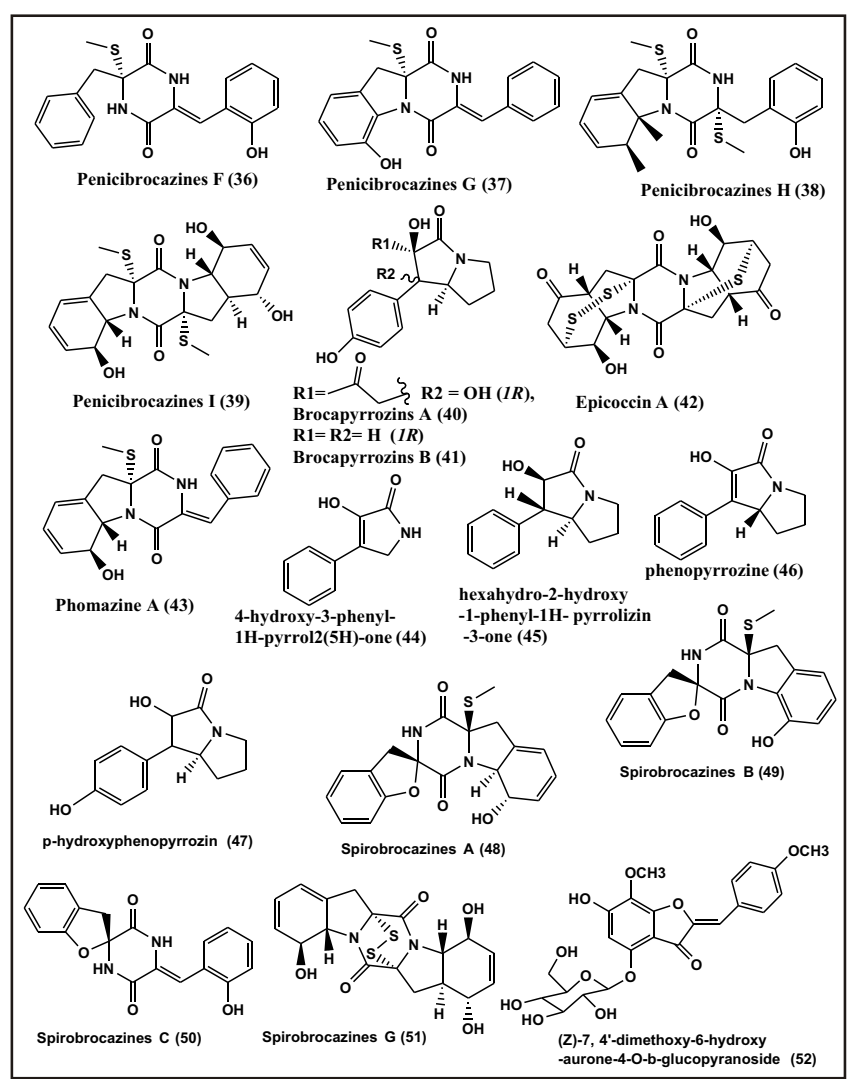

Fig. 4. Bio-actives reported from Penicillium sp. (36-52) an endophyte from $A$. marina 
Compound (51) also exhibited good antibacterial activity against $S$. aureus with MIC value of $0.25 \mu \mathrm{g} / \mathrm{mL}$, while positive control, chloromycetin displayed antibacterial activity with MIC, values of $0.5 \mu \mathrm{g} / \mathrm{mL}$. Compound (48) exhibited moderate activity with MIC values of 32.0, 16.0, and $64.0 \mu \mathrm{g} / \mathrm{mL}$ against E. coli, $S$. aureus, and $V$. harveyi, respectively while chloromycetin as positive control showed MIC values of $2.0,0.5$, and $2.0 \mu \mathrm{g} / \mathrm{mL}$, respectively. Compound (50) also exhibited activity against E. coli, Aeromonas hydrophilia, and $V$. harveyi, each with an MIC value of $32.0 \mu \mathrm{g} / \mathrm{mL}$. (Meng et al., 2016).

A new aurone glycoside, (Z)-7,4'-dimethoxy-6-hydroxyaurone-4-O-ß-glucopyranoside (52) (Fig. 5), was isolated from Penicillium sp.FJ-1, an endophyte associated with mangrove plant $A$. marina. Compound (52) displayed potent antifungal activity against Candida sp., comparable to that of amphotericin B and appeared better than fluconazole and also inhibited extracellular phospholipase secretion in a concentration-dependent manner (Song et al., 2015).

Two new compounds, named (Z)-7,4'-dimethoxy-6hydroxy-aurone-4-O-ß-glucopyranoside (DHAG) (52) and (-)-4-O-(4-O-ß-D-glucopyranosylcaffeoyl) quinic acid (53) (Fig. 5), were isolated from the endophytic fungus Penicillium citrinum of mangrove plant A. marina. Compound (53), exhibited potent chemoreversal activity, mainly by inhibiting P-glycoprotein efflux pump function (Liu et al., 2015a). It is reported that DHAG (52) increased the viability of PC12 cells, attenuated the imbalance of redox, and decreased cellular apoptosis in an $\mathrm{H}_{2} \mathrm{O}_{2}$-induced oxidative stress model. Furthermore, treatment with DHAG could markedly attenuate the anxiety-like behavior of rats induced by DOX. It is demonstrated that DHAG can be developed as a neuroprotective agent. (Li et al., 2019). DHAG (52) exerted anti-inflammatory effects by inhibiting inflammatory factors including the pro-inflammatory mediator NO and the proinflammatory cytokines IL-1b and TNF-a in LTA-stimulated H9c2 cells. Moreover, DHAG (52) considerably suppressed pro-inflammatory molecule production from upstream signaling pathways, which were involved in the progression of inflammatory responses in $\mathrm{H} 9 \mathrm{c} 2$ cells. Activation of NF-jB and MAPK leads to transcription factor binding to the promoter regions of pro-inflammatory cytokine genes, thereby enabling transduction of extracellular signals into cellular reactions (Dong et al., 2002). DHAG (52) decreased phosphorylation of several MAPKs, including JNK and P38, whose phosphorylation was induced by LTA stimulation. In addition, DHAG reduced nuclear translocation of NF-jB in response to LTA. It is also demonstrated that DHAG, LTA induced oxidative stress and inflammatory responses in cardiomyoblasts (Song et al., 2020).

Two new compounds, named as 4-(2',3'-dihydroxy-3'methyl-butanoxy)-phenethanol (54), and 15-hydroxy6a,12-epoxy-7ß,10aH,11ßH-spiroax-4-ene-12-one (55) (Fig. 5), were isolated from the endophytic fungus Penicillium sp.FJ-1 of mangrove A. marina collected in

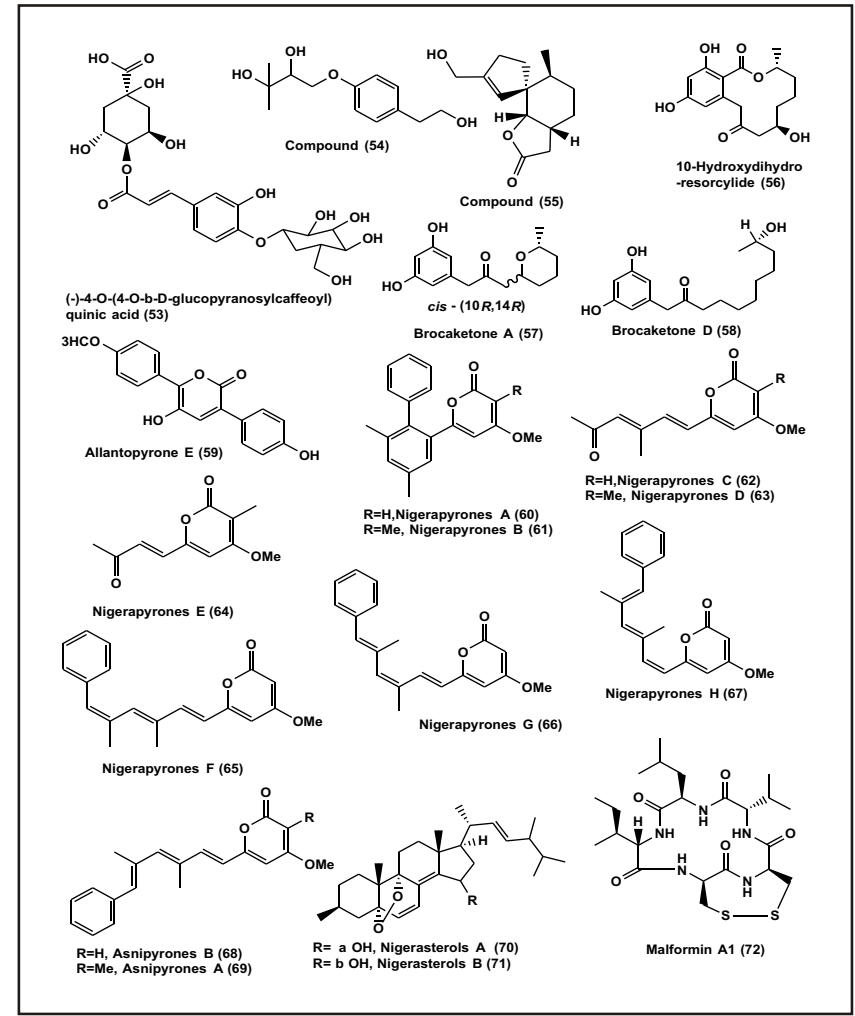

Fig. 5. Bio-actives reported from Penicillium sp. (53-58) and Aspergillus sp. (59-72) endophyte from $A$. marina

Fujian, China. Compound (55), displayed activity against Tca8113, MG-63 and WRL-68 cells with an $\mathrm{IC}_{50}$ value of 10 $\mu \mathrm{M}, 55 \mu \mathrm{M}$ and $58 \mu \mathrm{M}$, respectively. In nuce mice model, compound (55) displayed noteworthy inhibition of tumor growth of human osteosarcoma. Compound (54), displayed week activity with $\mathrm{IC}_{50}$ values of 26 and $35 \mu \mathrm{M}$, against Tca8113 and MG-63 cells, respectively. Positive control taxol, displayed cytototoxicity against Tca8113 and MG-63 cell lines with $\mathrm{IC}_{50}$ values of 46 and $10 \mathrm{nM}$, respectively (Zheng etal., 2014).

Compounds (10R,14R)-10-Hydroxydihydroresorcylide (56), brocaketone A (57) and brocaketone D (58) (Fig. 5), were purified from $P$. brocae MA-192, residing inside the leaves of $A$. marina, a mangrove plant, collected from Hainan Island, China. Compounds (56-58) displayed potent antioxidant activity in DPPH assay with $\mathrm{IC}_{50}$ values of 14.4 , 5.9 , and $16.3 \mu \mathrm{g} / \mathrm{mL}$, respectively, while positive control BHT displayed scavenging activity with $\mathrm{IC}_{50}$ value of $18.5 \mu \mathrm{g}$ /mL (Zhang et al., 2015).

\section{COMPOUNDS ISOLATED FROM ASPERGILLUS}

Anew a-pyrone derivative, Allantopyrone E (59) was purified from fungal endophyte $A$. versicolor associated with the fruit of the mangrove plant $A$. marina obtained from Port Safaga, Red Sea Governorate, Egypt. Allantopyrone E (59) displayed cytotoxic activity against HeLa cells with $\mathrm{IC}_{50}$ value of 50.97 $\mu \mathrm{M}$ (Elsbaey et al., 2020). 
Eight new a-pyrone derivatives, nigerapyrones A-B $(\mathbf{6 0}, \mathbf{6 1})$, (Fig. 5), C-E (62-64) and nigerapyrones F-H (65-67), together with previously reported congeners, asnipyrones B (68) and A (69) (Fig. 6), were purified from endophytic fungus $A$. niger MA-132, residing inside the fresh tissue of mangrove plant A. marina. Compound (64), displayed cytotoxic activity against SW1990, MDA-MB-231, A549, MCF- 7, HepG2, Du145, NCI-H460, and MDA-MB-231 cell lines with $\mathrm{IC}_{50}$ values of $38,48,43,105,86,86,43$, and $48 \mu \mathrm{M}$, respectively. Positive control, fluorouracil displayed cytotoxicity against A549, HepG2, DU145, MCF-7, SW1990, NCI-H460, and MDA-MB-231 cell lines, with IC $_{50}$ values of $52,109,3.3,31,121,8.5$, and $59 \mu \mathrm{M}$, respectively. Compound (61), was found selectively active against HepG2 cell line with an $\mathrm{IC}_{50}$ of $62 \mu \mathrm{M}$ while compound (69) exhibited activity against the $\mathrm{A} 549$ cell line with an $\mathrm{IC}_{50}$ of $62 \mu \mathrm{M}$, and nigerapyrone $\mathrm{D}(63)$ showed average or poor activity against the MCF-7, HepG2, and A549 cell lines, with $\mathrm{IC}_{50}$ values of 121, 81, and $81 \mu \mathrm{M}$, respectively (Liu et al., 2011).

Two novel sterols, nigerasterols A (70) and B (71), along with already reported cyclopentapeptides, malformins A1 (72) and C (73) (Fig. 6), were purified from A. niger MA-132, residing inside the mangrove plant $A$. marina. Compounds (70 and 71) displayed potent cytotoxic activity against HL60 cell line $\mathrm{IC}_{50}$ values of 0.30 and $1.50 \mu \mathrm{m}$, and against $\mathrm{A} 549$ cell line with $\mathrm{IC}_{50}$ values of 1.82 and $5.41 \mu \mathrm{m}$, respectively.

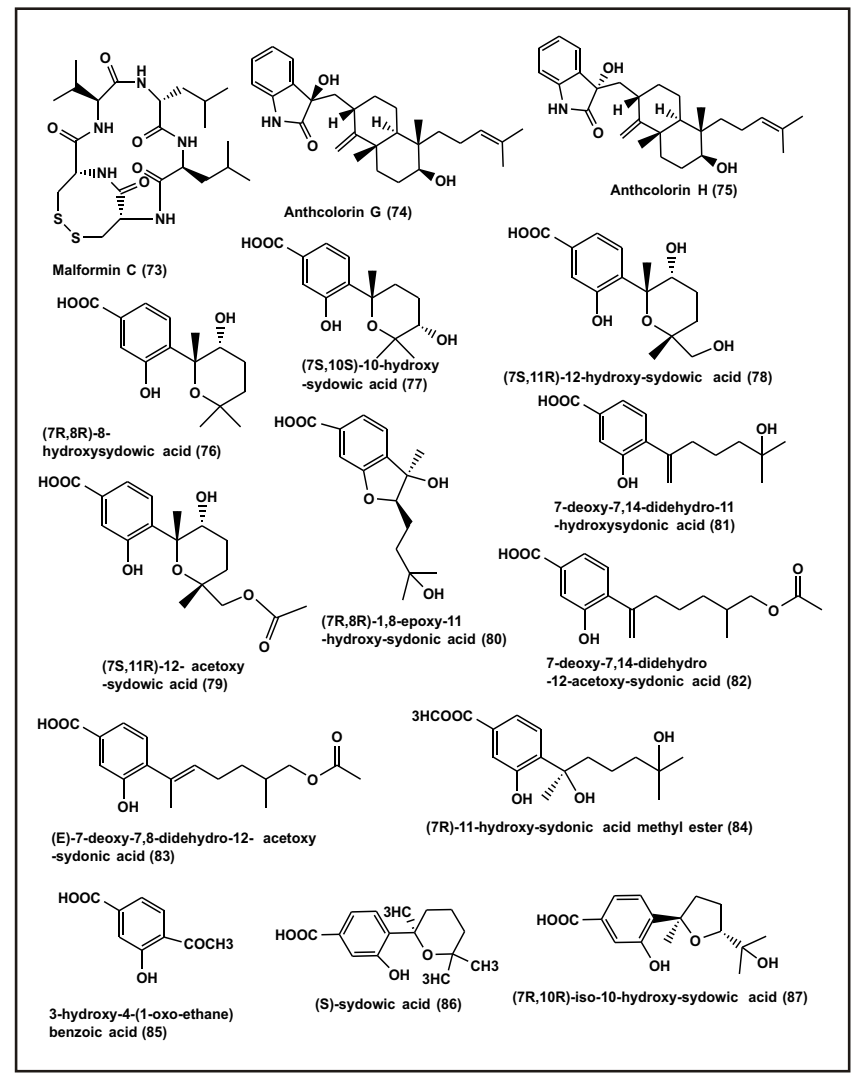

Fig. 6. Bio-actives reported from Aspergillus sp. (73-87) endophyte from $A$. marina
Compounds (72 and 73) displayed poor activity against Staphylococcus aureus with 9.0 and $8.5 \mathrm{~mm}$ diameter of clear zone on inhibition, respectively at a concentration of 20 $\mathrm{mg} /$ disk, while positive control chloramphenicol inhibited $S$. aureus with clear zone of inhibition of $20.0 \mathrm{~mm}$ at the same concentration (Liu etal., 2013).

Two new oxoindolo diterpene epimers, anthcolorin G (74) and anthcolorin $\mathrm{H}$ (75), nine new meroterpenes, (7R,8R)-8hydroxysydowic acid (76), (7S,10S)-10-hydroxy-sydowic acid (77), (7S,11R)-12-hydroxy-sydowic acid (78), (7S,11R)-12- acetoxy-sydowic acid (79), (7R,8R)-1,8epoxy-11-hydroxy-sydonic acid (80), 7-deoxy7,14-didehydro -11-hydroxysydonic acid (81), 7-deoxy7,14-didehydro-12-acetoxy-sydonic acid (82) (Fig. 6), and (E)-7-deoxy-7,8-didehydro-12-acetoxy-sydonic acid (83), (7R)-11-hydroxy-sydonic acid methyl ester (84), and a benzoic acid derivative, 3-hydroxy-4-(1-oxo-ethane) benzoic acid (85), in addition to twelve known compounds (S)sydowic acid (86), (7R,10R)-iso-10-hydroxy-sydowic acid (87), engyodontiumone J (88), engyodontiumone I (89), (E)-7-deoxy-7,8-didehydro12-hydroxy-sydonic acid (90), (7R)-11-hydroxysydonic acid (91), an epimeric mixture of (7R,11R) and (7R,11S)-12-acetoxy sydonic acid (92), 12acetoxy-1-deoxy-sydonic (93), diorcinol (94), macrosporin (95), and ergostrol peroxide (96) (Fig. 7), were purified from endophytic fungus Aspergillus versicolor isolated from mangrove plant $A$. marina and grown on the solid rice culture. The site of collection was $17 \mathrm{~K}$ Safaga, Red Sea, Egypt.

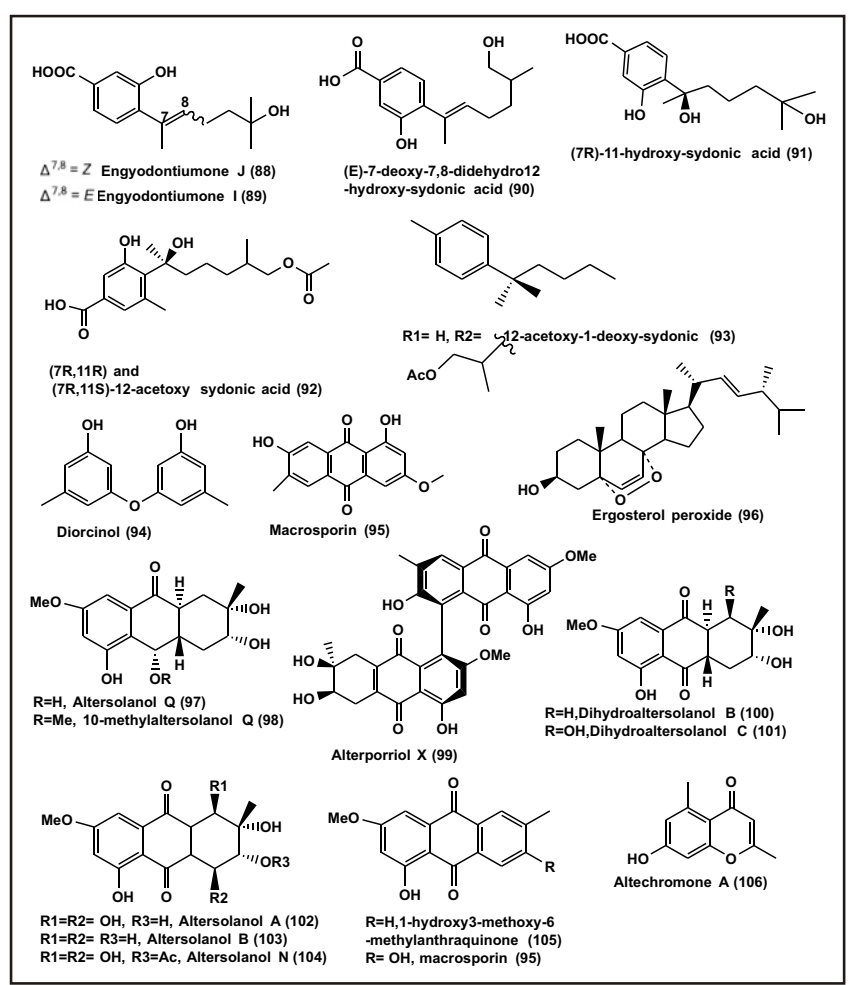

Fig. 7. Bio-actives reported from Aspergillus sp. (87-96) sand Stemphylium sp. (97-106) endophyte from $A$. marina 
Compounds $\left(\mathbf{7 5}, \mathbf{8 2}, \mathbf{8 3}\right.$ and 94), were found active with $\mathrm{IC}_{50}$ values of $43.7,83.8,53.5,83.8 \mu \mathrm{M}$, respectively against Hela cell lines (Elsbaey et al., 2019).

\section{COMPOUNDS ISOLATED FROM OTHER ENDOPHYTIC FUNGI}

Two new anthraquinones, altersolanol Q (97) and 10methylaltersolanol Q (98), and the new dimer alterporriol X (99), along with 13 known analogs dihydroaltersolanol B (100) and C (101) (Fig.7), altersolanol A (102), B (103), and $\mathrm{N}$ (104), 1-hydroxy3-methoxy-6-methylanthraquinone (105), macrosporin (95), altechromone A(106), alterporriol D (107), E (108), R (109), V (110), and W (111) (Fig. 8), were extracted from endophytic fungus $S$. globuliferum grown on white bean solid culture media. S. globuliferum, was purified from the Egyptian mangrove plant $A$. marina (Moussa et al., 2016). Compounds (101-103 and 108), were found active against L5178Y cell lines with $\mathrm{IC}_{50}$ values of 3.4, 2.53, 3.78, and 6.9, $\mu \mathrm{M}$, respectively (Debbab et al., 2009; Liu et al., 2015b). Compound (104) also exhibited potent cytotoxicity against L5178Y cells with $\mathrm{IC}_{50}$ values in the low micro-molar range (Debbab et al., 2012). Mishra et al. (2015) reported that Compound (102) displayed potent cytotoxicity against 34 human cancer cell lines in vitro, with mean $\mathrm{IC}_{50}$ values of $0.005 \mu \mathrm{g} / \mathrm{mL}$ (Mishra et al., 2015).

Five novel 12-membered macrolides containing thioethers, thiocladospolides F-J (112-116), along with previously isolated analogues, pandangolide 3 (117), thiocladospolide A (118), seco-secopatulolide C (119), and iso-cladospolide B (120) (Fig. 8), were isolated from endophytic fungus Cladosporium oxysporum associated with the root of

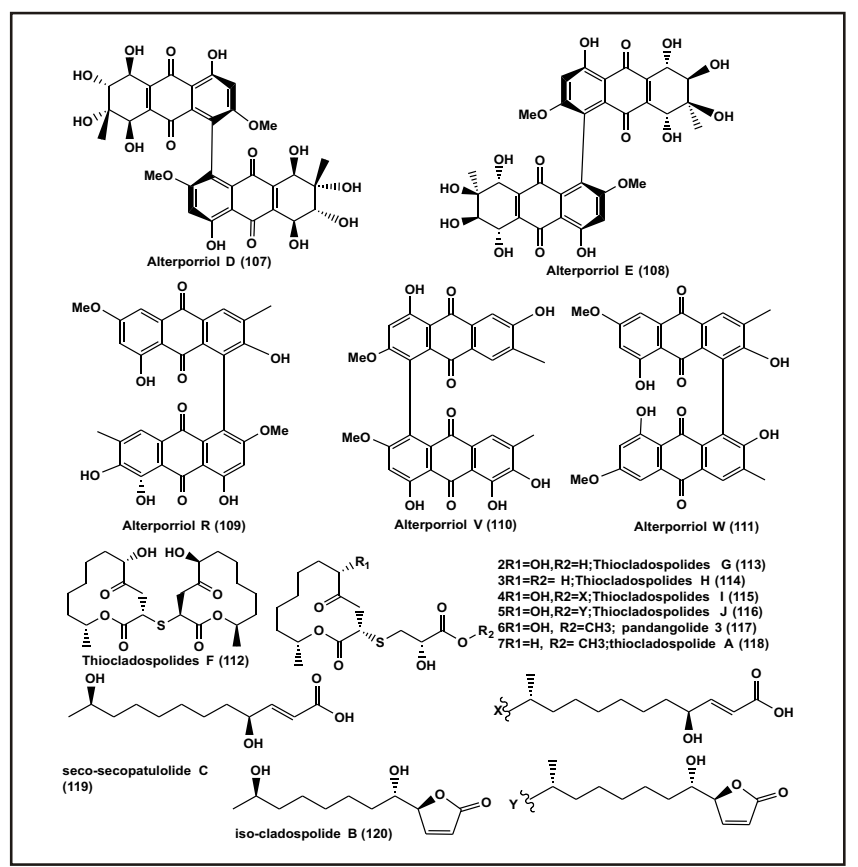

Fig. 8. Bio-actives reported from Stemphylium sp. (107 111) and Cladosporium sp. (112-120) endophyte from A. marina mangrove plant $A$. marina collected from Hainan Province, China. Compounds (112-120) displayed antibacterial activity with MIC values ranging from 4 to $32 \mu \mathrm{g} / \mathrm{mL}$ against Edwardsiella tarda and E. ictarda, the aquatic pathogens. Compound (113) exhibited potent activity against E. tarda with MIC values of $4 \mu \mathrm{g} / \mathrm{mL}$ and compound (120) was found active against plant pathogenic fungus Cytospora mandshurica with MIC values of $8 \mu \mathrm{g} / \mathrm{mL}$ (Wang et al., 2020).

A new lactone, 1,8-dihydroxy-10-methoxy-3-methyldibenzo [b,e]oxepine-6,11-dione (121), and two new xanthones, 1hydroxy-8-(hydroxymethyl)-6-methoxy-3-methyl-9Hxanthen-9-one (122) and 1-hydroxy-8-(hydroxymethyl)-3methoxy-6-methyl-9H-xanthen -9-one (123) (Fig. 9) were purified from Phoma sp. SK3RW1M residing inside the roots of $A$. marina. The site of collection was Shankou mangrove, Guangxi, China. Compounds (121-123) were inactive when tested for cytotoxic properties (Pan et al., 2010).

Two new metabolites, the cyclo-(L-Phe-L-Leu1-L-Leu2-LLeu3-L-Ile) (124) and (3S,4R)-dihydroxy-(6S)-undecyl-apyranone (125) (Fig. 3) were purified from endophytic fungus number 2524 residing inside the seed of $A$. marina collected from Hong Kong. Both the compounds displayed poor activity against Bel-7402, NCI-4460 cancer cell line and L-02 the normal human cell lines (Li et al., 2004). Two new metabolites, namely 2106 A (126) and cyclo-(N-MeVal-NMeAla) (127) (Fig. 3) were obtained from endophytic fungus number 2106 isolated from the seeds of the mangrove $A$. marina in Hong Kong. No activity is reported for both the compounds (Wang et al., 2008).

A known farinomalein derivative Farinomalein (128) along

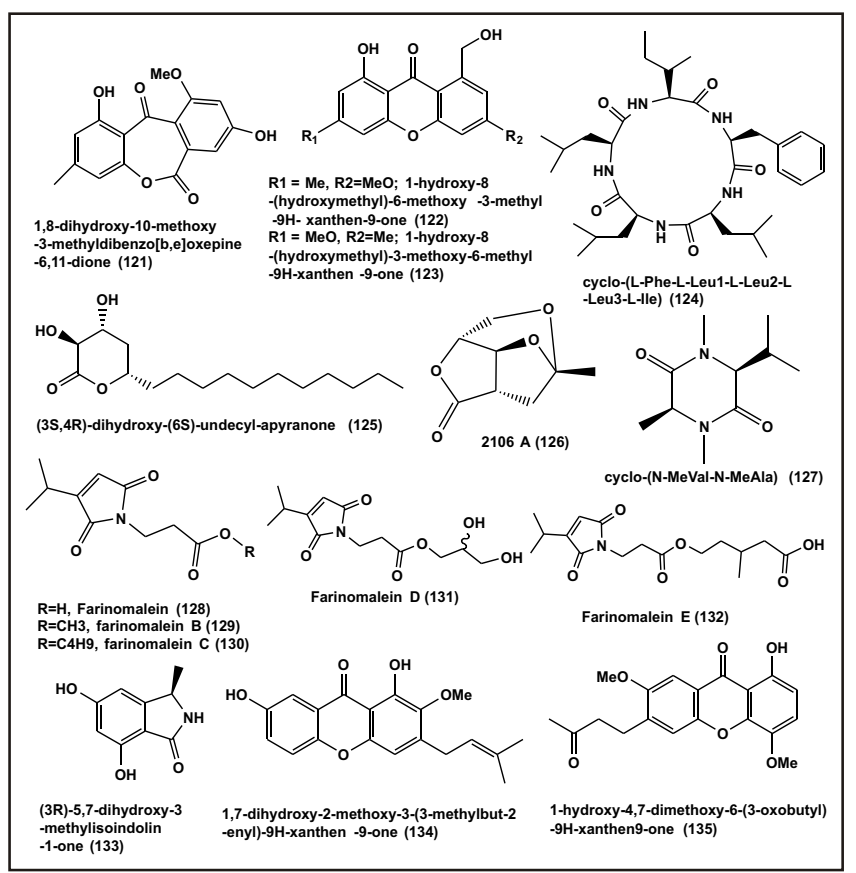

Fig.9. Bio-actives reported from Phoma sp. (121-123) and Unidentified endophytes (124-135) from A. marina 
with three new farinomalein derivatives, farinomaleins B-E (129-132), and one new isoindoline congener (3R)-5,7dihydroxy-3-methylisoindolin-1-one. (133) (Fig. 9), were extracted from an unidentified endophytic fungus residing inside the inner tissues of plant $A$. marina from Oman. Only compound (129) displayed average cytotoxicity against L5178Y cells with $\mathrm{IC}_{50}$ value of $4.4 \mu \mathrm{g} / \mathrm{mL}$ (El Amrani et al., 2012).

Two new xanthone derivatives, 1,7-dihydroxy-2-methoxy-3(3-methylbut-2-enyl)-9H -xanthen-9-one (134) and 1hydroxy-4,7-dimethoxy-6-(3-oxobutyl) -9H-xanthen9-one (135) (Fig. 9), were purified from the mangrove endophytic fungus (No. ZH19) residing inside the leaves of $A$. marina from the Dong Sai of the South China Sea coast. Compounds (134) displayed cytotoxicity against KB and KBV200 cells with $\mathrm{IC}_{50}$ values of 20 and $30 \mu \mathrm{M}$ while compound (135) was found active against both the cells with $\mathrm{IC}_{50}$ values of 35 and $41 \mu \mathrm{M}$, respectively (Huang et al., 2010).

\section{AN OVERVIEW AND CONCLUSION AND FUTURE PROSPECTS}

In the present study, we have reported 135 compounds from mangrove plant $A$. marina with various biological activities (antibacterial, antifungal, anticancer, anti-inflammatory, antioxidant, anti-angiogenesis, and L-calcium channel blocker activity). These compounds belong to the various chemical classes such as anthraquinone, piperazine, glycoside, cyclopeptides, sterol, ergostrol, xanthone, macrolide, etc. (Table 2). Some of the isolated compounds, viz. (Z)-7,4'-dimethoxy-6-hydroxy-aurone-4-O- $\beta$ glucopyranoside (DHAG) (52) with potent antiinflammatory, altersolanol A (102) with anticancer, and Xyloketal B (2) with neuroprotective activity can be potential drug candidates. In this review we found majority of the compounds were isolated from the endophytic fungal genera Xylaria, Aspergillus, Penicillium, Stemphylium, Cladosporium, Phoma and an Unidentified fungus. It is also found that only a few compounds were screened for biological activity due to insufficient quantity, hence there is a need to produce the compounds in sufficient quantities and to evaluate these compounds in various screening activities using high throughput screening.

The methods like OSMAC, Co -cultivation can help in exploring chemical diversity. The application of epigenetic modifiers in culture media will help in expressing the biosynthetic gene clusters (BGC), responsible for unexpressed bioactive metabolites hence increasing chemical diversity. Next-generation sequencing (NSG) data in combination with other bioinformatics tools will help in generating chemical diversity. Around a dozen secondary metabolites were reported from 14 fungal species out of 149 marine fungi screened for secondary metabolites from mangrove plant $A$. marina (Abdel-Wahab et al., 2020). It is advisable to explore the fungal diversity from different locations for bioactive metabolites as the factors like salinity, temperature, the maturity of the mangrove site, availability of
Table2. Chemical class of various metabolites identified from Endophytic fungi of Avicennia marina

\begin{tabular}{|l|l|c|l|}
\hline \multicolumn{1}{|c|}{ Species } & \multicolumn{1}{|c|}{ Class } & Metabolite & \multicolumn{1}{c|}{ Reference } \\
\hline Xylaria sp. (no. 2508) & Phenolic & 10 & Lin et al., 2001a \\
\hline Xylaria sp. (no. 2508) & Allenic ethers & $11-13$ & Lin et al., 2001b \\
\hline Xylaria sp. (no. 2508) & Benzofuran & 17 & Xu et al., 2008 \\
\hline Xylaria sp. (2508) & Pyridine & 18 & Xu et al., 2009 \\
\hline Penicillium brocae MA-231 & Pyran & 21,22 & Meng et al., 2015a \\
\hline P. Brocae ma-231 & Piperazine & $23-27$ & Meng et al., 2015b \\
\hline P. Brocae ma-231 & Piperazine & $29-34$ & Meng et al., 2014 \\
\hline P. Brocae ma-231 & Piperazine & $36-39$ & Meng et al., 2017 \\
\hline P. Brocae ma-231 & Pyrrozin & 40,41 & Meng et al., 2017 \\
\hline P. Brocae ma-231 & Pyrrol & 44 & Meng et al., 2017 \\
\hline P. Brocae ma-231 & Piperazines & $48-51$ & Meng et al., 2016 \\
\hline Penicillium sp.FJ-1 & Glycoside & 52 & Song et al., 2015 \\
\hline Penicillium citrinum & Glycoside & 53 & Song et al., 2020 \\
\hline Aspergillus versicolor & Pyrone & 59 & Elsbaeyet al.,2020 \\
\hline Aspergillus niger MA-132 & Pyrone & $60-69$ & Liu et al., 2011 \\
\hline A. Niger ma-132 & Sterol & 70,71 & Liu et al., 2013 \\
\hline A. Niger ma-132 & Cyclopeptides & 72,73 & Liu et al., 2013 \\
\hline Aspergillus versicolor & Terpene & $74-83$ & Elsbaey et al., 2019 \\
\hline Aspergillus versicolor & Benzoic acid & 85 & Elsbaey et al., 2019 \\
\hline Aspergillus versicolor & Sydowic acid & 86,87 & Elsbaey et al., 2019 \\
\hline Aspergillus versicolor & Sydonic acid & $90-93$ & Elsbaey et al., 2019 \\
\hline Aspergillus versicolor & Ergostrol & 96 & Elsbaey et al., 2019 \\
\hline Stemphylium globuliferum & Solanol & $97,98,100-104$ & Moussa et al., 2016 \\
\hline Stemphylium globuliferum & Anthraquinone & 105 & Moussa et al., 2016 \\
\hline Cladosporium oxysporum & Macrolide & $112-116$ & Wang et al., 2020 \\
\hline Phoma sp. SK3RW1M & Lactone & 121 & Pan et al., 2010 \\
\hline & & & \\
\hline
\end{tabular}

decaying materials inside the mangroves, associated mangrove and terrestrial trees, tidal amplitude, and other factors account for this. The fact that typical marine fungi grow slowly, and their maintenance is difficult and hence many of them are not screened for bioactives, made mycologists to look for the isolation of endophytic fungi which are relatively easier to isolate and maintain, are the ones screened more for bioactive compounds.

Mangrove plant $A$. marina is used in various traditional and folk medicines and is known to produce diverse chemical compounds (El Dohaji et al., 2020). The endophytes and other marine fungi associated with this plant can be a potential source of bioactive metabolites and should be the prime target in the exploration of new drugs due to their application in the pharmaceutical industry.

\section{REFERENCES}

Abdel-Wahab, M.A., Jones, E.B.G. and Bahkali, A.H. 2020. Marine fungi recorded from Avicennia marina (Forsk.) Vierh. and their secondary product potential. Nova Hedwigia 111: 357-390.

Chen, L., Chaichana, K.L., Kleinberg, L., Ye, X., QuinonesHinojosa, A. and Redmond, K. 2015. Glioblastoma recurrence patterns near neural stem cell regions. Radiother. Oncol. 116 (2): 294-300.

Debbab, A., Aly, A.H., Edrada-Ebel, R., Wray, V., Müller, W.E., Totzke, F., Zirrgiebel, U., Schachtele, C., 
Kubbutat, M.H., Lin, W.H. and Mosaddak, M. 2009. Bioactive metabolites from the endophytic fungus Stemphylium globuliferum isolated from Mentha pulegium.J. Nat. Prod. 72(4): 626-631.

Debbab, A., Aly, A.H., Edrada-Ebel, R., Wray, V., Pretsch, A., Pescitelli, G., Kurtan, T. and Proksch, P. 2012. New anthracene derivatives-structure elucidation and antimicrobial activity. Eur. J. Org. Chem. 2012 (7): 1351-1359.

Deshmukh, S. K., Agrawala, S., Prakash, V., Reddy, M.S.and Gupta, M. K. 2020. Anti-infectives from mangrove endophytic fungi. S. Afr. J. Bot. 134: 237-267.

Deshmukh, S. K., Gupta, M. K. Prakash, V. and Reddy, M.S. 2018. Mangrove-associated fungi a novel source of potential anticancer compounds. J. Fungi. 4: 101, doi:10.3390/jof4030101

Deshmukh, S.K., Verekar, S.A. and Bhave, S. 2015. Endophytic fungi: an untapped source for antibacterials. Front. Microbiol., Https://doi.org/ 10.3389/fmicb.2014.00715

Dong, C, Davis, R.J. and Flavell, R.A. 2002. MAP Kinases in the immune response. Annu. Rev. Immunol. 20: 5572 .

Duke, N.C., 1991. A systematic revision of the mangrove genus Avicennia (Avicenniaceae) in Australasia. Aust. Syst. Bot. 4 (2): 299-324.

El Amrani, M., Debbab, A., Aly, A.H., Wray, V., Dobretsov, S., Müller, W.E., Lin, W., Lai, D. and Proksch, P. 2012. Farinomalein derivatives from an unidentified endophytic fungus isolated from the mangrove plant Avicennia marina. Tetrahedron Lett. 53 (49): 6721 6724.

El Dohaji, L.M., Hamoda, A.M., Hamdy, R. and Soliman, S.S. 2020. Avicennia marina a natural reservoir of phytopharmaceuticals: Curative power and platform of medicines. J. Ethnopharmacol. 263: 113179. DOI: 10.1016/j.Jep. 2020.113179

Elsbaey, M., Tanaka, C. and Miyamoto, T. 2020. Allantopyrone E, a rare a-pyrone metabolite from the mangrove derived fungus Aspergillus versicolor. Nat. Prod. Res., pp .1-5. DOI: 10.1080/14786419.2020.1803309

Elsbaey, M., Tanaka, C. and Miyamoto, T. 2019. New secondary metabolites from the mangrove endophytic fungus Aspergillus versicolor. Phytochem. Lett. 32: 70-76.

Huang, Z., Yang, R., Yin, X., She, Z. and Lin, Y., 2010. Structure elucidation and NMR assignments for two xanthone derivatives from a mangrove endophytic fungus (No. ZH19). Magnetic Resonance in Chemistry 48 (1): 80-82.

Kumaresan, V. and Suryanarayanan, T. 2001. Occurrence and distribution of endophytic fungi in a mangrove community. Mycol. Res. 105: 1388-1391.
Li, H., Li, X., Luo, W. and Zhu, X. 2019. (Z)-7, 4'Dimethoxy-6-hydroxy-aurone-4 - O- $\beta$ glucopyranoside exerts neuroprotective effects in vitro and anxiolytic activity in vivo. Neuroreport 30 (4): $280-287$

Li, H.J., Lin, Y.C., Yao, J.H., Vrijmoed, L.L.P. and Jones, E.G. 2004. Two new metabolites from the mangrove endophytic fungus No. 2524. J. Asian Nat. Prod. Res. 6(3): 185-191.

Lin, Y., Wu, X., Feng, S., Jiang, G., Luo, J., Zhou, S., Vrijmoed, L.L.P., Jones, E.G., Krohn, K., Steingrover, K. and Zsila, F. 2001a. Five unique compounds: xyloketals from mangrove fungus Xylaria sp. from the South China Sea coast. J. Org. Chem. 66 (19): 6252-6256.

Lin, Y., Wu, X., Feng, S., Jiang, G., Zhou, S., Vrijmoed, L.L.P. and Jones, E.G. $2001 \mathrm{~b}$. A novel Ncinnamoylcyclopeptide containing an allenic ether from the fungus Xylaria sp.(strain\# 2508) from the South China Sea. Tetrahedron Lett. 42 (3): 449-451.

Liu, J., Xu, M., Zhu, M.Y. and Feng, Y., 2015a. Chemoreversal metabolites from the endophytic fungus Penicillium citrinum isolated from a mangrove Avicennia marina. Nat. Prod. Commun. 10 (7), p.1934578X1501000717.DOI:10.1177/ $1934578 \times 1501000717$.

Liu, Y., Marmann, A., Abdel-Aziz, M.S., Wang, C.Y., Müller, W.E., Lin, W.H., Mándi, A., Kurtán, T., Daletos, G. and Proksch, P. 2015b. Tetrahydroanthraquinone derivatives from the endophytic fungus Stemphylium globuliferum. Eur. J. Org. Chem. 2015 (12): 2646-2653.

Liu, D., Li, X.M., Li, C.S. and Wang, B.G. 2013. Nigerasterols A and B, antiproliferative sterols from the Mangrove-Derived Endophytic Fungus Aspergillus niger MA-132. Helv. Chim. Acta. 96 (6): 1055-1061.

Liu, D., Li, X.M., Meng, L., Li, C.S., Gao, S.S., Shang, Z., Proksch, P., Huang, C.G. and Wang, B.G. 2011. Nigerapyrones A-H, a-pyrone derivatives from the marine mangrove -derived endophytic fungus Aspergillus niger MA-132. J. Nat. Prod. 74 (8): 1787-1791.

Liu, X., Xu, F., Zhang, Y., Liu, L., Huang, H., Cai, X., Lin, Y. and Chan, W. 2006. Xyloketal H from the mangrove endophytic fungus Xylaria sp. 2508. Russ. Chem. Bull. 55 (6): 1091-1092.

Lu, X. L., Xu, Z. L., Yao, X. L., Su, F. J., Ye, C. H., Li, J., Lin, Y.C., Wang, G.L., Zeng, J.S., Huang, R.X. and Ou, J.S. 2012. Marine cyclotripeptide X-13 promotes angiogenesis in zebrafish and human endothelial cells via PI3K/Akt/eNOS signaling pathways. Mar. Drugs 10 (6): 1307-1320.

Meng, L.H., Li, X.M., Liu, Y., Xu, G.M. and Wang, B.G. 2017. Antimicrobial alkaloids produced by the 
mangrove endophyte Penicillium brocae MA-231 using the OSMAC approach. RSC advances 7 (87): 55026-55033.

Meng, L.H., Wang, C.Y., Mandi, A., Li, X.M., Hu, X.Y., Kassack, M.U., Kurtan, T. and Wang, B.G. 2016. Three diketopiperazine alkaloids with spirocyclic skeletons and one bisthiodiketopiperazine derivative from the mangrove-derived endophytic fungus Penicillium brocae MA-231. Org. Lett. 18: 5304-5307.

Meng, L.H., Li, X.M., Liu, Y. and Wang, B.G. 2015a. Polyoxygenated dihydropyrano [2,3-c] pyrrole-4, 5 -dione derivatives from the marine mangrovederived endophytic fungus Penicillium brocae MA231 and their antimicrobial activity. Chin. Chem. Lett. 26 (5): 610-612.

Meng, L.H., Zhang, P., Li, X.M. and Wang, B.G. $2015 \mathrm{~b}$. Penicibrocazines A-E, five new sulfide diketopiperazines from the marine-derived endophytic fungus Penicillium brocae. Mar. drugs 13 (1): 276-287.

Meng, L.H., Li, X.M., Lv, C.T., Huang, C.G. and Wang, B.G. 2014. Brocazines A-F, cytotoxic bisthiodiketopiperazine derivatives from Penicillium brocae MA-231, an endophytic fungus derived from the marine mangrove plant Avicennia marina. J. Nat. Prod. 77 (8): 1921-1927.

Mishra, P.D., Verekar, S.A., Deshmukh, S.K., Joshi, K.S., Fiebig, H.H. and Kelter, G. 2015. Altersolanol A: A selective cytotoxic anthraquinone froma Phomopsis sp. Lett. Appl. Microbiol. 60:387-391.

Moussa, M., Ebrahim, W., El-Neketi, M., Mandi, A., Kurtan, T., Hartmann, R., Lin, W., Liu, Z.and Proksch, P. 2016. Tetrahydroanthraquinone derivatives from the mangrove-derived endophytic fungus Stemphylium globuliferum. Tetrahedron Lett. 57: 4074-4078

Pan, J.H., Deng, J.J., Chen, Y.G., Gao, J.P., Lin, Y.C., She, Z.G. and Gu, Y.C. 2010. New lactone and xanthone derivatives produced by a mangrove endophytic fungus Phoma sp. SK3RW1M from the South China Sea. Helv. Chim. Acta. 93 (7): 1369-1374.

Rashmi, M., Kushveer, J.S. and Sarma, V.V. 2019. A worldwide list of endophytic fungi with notes on ecology and diversity. Mycosphere 10: 798-1079.

Song, Q., Xie, X., Hu, Z., Xue, J., Zhang, S. and Xie, X., 2020. (Z)-7, 4'-dimethoxy-6-hydroxy-aurone-4-O- 3 glucopyranoside attenuates lipoteichoic acidinduced damage in rat cardiomyoblast cells. J. Int. Med. Res. 48 (8): p.0300060519889716.

Song, Y.X., Ma, Q. and Li, J., 2015. A new aurone glycoside with antifungal activity from marine-derived fungus Penicillium sp. FJ-1. Zhong Yao Cai 40 (6): 1097-1101.

Tomlinson, P. B. 1986. Avicenniaceae. In: The Botany of Mangroves. Cambridge Univ. Press pp. 186-207
Wang, W., Feng, H., Sun, C., Che, Q., Zhang, G., Zhu, T. and Li, D. 2020. Thiocladospolides FJ, antibacterial sulfur containing 12-membered macrolides from the mangrove endophytic fungus Cladosporium oxysporum HDN13-314. Phytochemistry 178: p.112462.

Wang, S.Y., Xu, Z.L., She, Z.G., Wang, H., Li, C.R. and Lin, Y.C. 2008. Two new metabolites from the mangrove endophytic fungus no. 2106. J. Asian Nat. Prod. Res. 10 (7): 622-626.

Wu, X.Y., Liu, X.H., Lin, Y.C., Luo, J.H., She, Z.G., Houjin, L., Chan, W.L., Antus, S., Kurtan, T., Elsasser, B. and Krohn, K. 2005a. Xyloketal F: A strong Lcalcium channel blocker from the mangrove fungus Xylaria sp. (\# 2508) from the South China Sea Coast. Eur J. Org. Chem. 2005 (19): 4061-4064.

Wu, X., Liu, X., Jiang, G., Lin, Y., Chan, W. and Vrijmoed, L.L.P. 2005b. Xyloketal G, a Novel Metabolite from the Mangrove Fungus Xylaria sp. 2508. Chem. Nat. Compd. 41 (1): 27-29.

Xiao, A.J., Chen, W., Xu, B., Liu, R., Turlova, E., Barszczyk, A., Sun, C.L., Liu, L., Deurloo, M., Wang, G.L. and Feng, Z.P. 2015. Marine compound xyloketal B reduces neonatal hypoxic-ischemic brain injury. Mar. Drugs 13(1):.29-47.

Xu, F., Pang, J., Lu, B., Wang, J., Zhang, Y., She, Z., Vrijmoed, L.L.P., Gareth Jones, E.B. and Lin, Y. 2009. Two metabolites with DNA-binding affinity from the mangrove fungus Xylaria sp. (\# 2508) from the South China Sea Coast. Chin. J. Chem. 27(2):365-368.

Xu, F., Zhang, Y., Wang, J., Pang, J., Huang, C., Wu, X., She, Z., Vrijmoed, L.L.P., Jones, E.G. and Lin, Y. 2008. Benzofuran derivatives from the mangrove endophytic fungus Xylaria sp.(\#2508). J. Nat. Prod. 71 (7): 1251-1253.

Yin, W., Lin, Y., She, Z., Vrijmoed, L.L.P. and Jones, E.G. 2008. A new compound: Xyloketal H from mangrove fungus Xylaria sp. from the South China Sea Coast. Chem. Nat. Compd. 44 (1): 3-5.

Zhang, P., Meng, L. H., Mándi, A., Li, X. M., Kurtán, T. and Wang, B. G. 2015. Structure, absolute configuration, and conformational study of resorcylic acid derivatives and related congeners from the fungus Penicillium brocae. RSC Advances 5: 39870-39877.

Zheng, C., Chen, Y., Jiang, L.L. and Shi, X.M., 2014. Antiproliferative metabolites from the endophytic fungus Penicillium sp. FJ-1 isolated from a mangrove Avicennia marina. Phytochem. Lett. 10: 272-275.

Zhu, F., Chen, X., Yuan, Y., Huang, M., Sun, H. and Xiang, W., 2009. The chemical investigations of the mangrove plant Avicennia marina and its endophytes. Open Nat. Prod. J. 2(1): 24-32. 